\title{
Transforming growth factor- $\beta 3$ induced rat bone marrow-derived mesenchymal stem cells differentiation into smooth muscle cells by activating Myocardin
}

\author{
Lin-Lin Ma ${ }^{1,2}$, Nan Wang ${ }^{1,2}$, Zhen Zhou ${ }^{1,2}$, Jun-Yun Zhang ${ }^{1,2}$, Xue-Gang Luo ${ }^{1,2}$, Yong Jiang ${ }^{1,2}$, \\ Tong-Cun Zhang ${ }^{1,2^{*}}$
}

${ }^{1}$ Key Laboratory of Industrial Microbiology, Ministry of Education, Tianjin, China;
${ }^{2}$ College of Biotechnology, Tianjin University of Science and Technology, Tianjin, China.
Email: hippopotamus19851010@126.com; ${ }^{*}$ tony@tust.edu.cn

Received 10 July 2009; revised 21 August 2009; accepted 31 August 2009.

\begin{abstract}
Bone marrow mesenchymal stem cells (MSCs) can differentiate into smooth muscle cells (SMCs) and have tremendous potential for cell therapy and tissue engineering. In this study, to understand the effects of TGF- $\beta 3$ on rat bone marrow-derived MSCs and the underlying molecular mechanism of this differentiation process, we investigated that the changes of myocardin-related transcription factors (MRTFs) at the transcriptional level after rat MSCs were treated with TGF- $\beta 3$. The results showed that TGF- $\beta 3$ increased the expression of contractile genes, such as SM22, smooth muscle-myosin heavy chain (SM- MHC), SM- $\alpha$-actin in MSCs. When TGF- $\beta 3$ induced MSCs differentiation into SMCs, myocardin and MRTF-A were activated. The data indicated that TGF- $\beta 3$ induced rat bone marrow-derived MSCs differentiation into SMCs by activating mypcardin and MRTF-A.
\end{abstract}

Keywords: Mesenchymal Stem Cells; Smooth Muscle Cells; TGF- $\beta$; MRTFs

\section{INTRODUCTION}

In vivo, smooth muscle cells (SMCs) are found in the vascular system, as well as in visceral organs, notably the respiratory, genitourinary, and gastrointestinal systems. VSMCs were involved in atherosclerosis and hypertension, leading causes of heart failure. Thus there was the great interest in the field of cellular therapeutics involving these tissues. However, one major limitation to this approach has been that a reliable source of SMCs can be impractical and morbid. In addition, biopsies

This work was financially supported by National Natural Science Foundation of China (No.30800561), Tianjin Natural Science Foundation (09JCZDJC18100) and Scientific Research Foundation of Tianjin University of Science and Technology (20080409). usually lead to limited amounts of cells. It has been shown that SMCs derived from diseased organs can lead to abnormal cells that are different from healthy SMCs [1]. Therefore, there is a great need for alternative sour ces of healthy SMCs.

Several groups have suggested the use of bone marrow-derived cells to repair smooth muscle tissues because of their stem cell-like properties [2]. Bone marrow-derived mesenchymal stem cells (MSCs) have a self-renewal capacity, long-term viability. It is important that MSCs can differentiate into a variety of cell types, such as osteogenic, adipogenic, chondrogenic, skeletal muscle cells, and SMCs in response to different microenviromental cues [3]. In vivo, MSCs transplanted into the heart can differentiate into SMCs and contribute to the remodeling of vasculature [4]. These findings indicated that MSCs might be as sources of healthy SMCs under some specific conditions, such as in the presence of some cytokine.

Transforming growth factor- $\beta$ (TGF- $\beta$ ) proteins are multifunctional proteins that regulate cell growth, differentiation, migration, and extracellular matrix production. It has been shown recently that TGF- $\beta$ increases smooth muscle (SM)-actin expression in MSCs [5]. Sphingosylphosphorylcholine (SPC) induces differentiation of human adipose-tissue-derived MSCs into smooth-muscle-like cells through a TGF- $\beta$-dependent mechanism [6]. These results indicate that the TGF- $\beta$ was involved in mesenchymal lineage cell type differentiation into SMCs. Therefore, we investigated the role of TGF- $\beta$ in bone marrow-derived MSC differentiation into SMCs.

Myocardin is expressed specifically in smooth and cardiac muscle cell lineages and activates smooth and cardiac muscle reporter genes by interacting with serum response factor (SRF). Myocardin shares homology with myocardin-related transcription factor-A (MRTF-A), and MRTF-B, which are expressed in a broad range of embryonic and adult tissues [7]. To understand whether MRTFs are implicated in the SMC differentiation of 
MSCs, we investigated the changes of MRTF family mRNA level after MSCs were induced by TGF- $\beta 3$.

In this report, we show that TGF- $\beta 3$ induced rat bone marrow-derived MSC differentiation into SMCs. TGF$\beta 3$ increased the expression of contractile genes, such as SM22, smooth muscle-myosin heavy chain (SM-MHC), SM- $\alpha$-actin in MSCs. We also demonstrated that myocardin and MRTF-A play important roles in the TGF$\beta 3$-induced SMC differentiation in MSCs.

\section{MATERIALS AND METHODS}

\subsection{Reagents}

Dulbecco's modified Eagle's medium-low glucose (DMEM-LG) was purchased from Hyclone Co. TGF- $\beta 3$ was purchased from Peoro Tech. Co. Fetal bovine serum (FBS) was obtained from Hycolon Lab, Inc. Fluorescein isothiocyanate (FITC)-conjugated or phycoerythrin (PE)conjugated antibodies CD14, CD29, CD34, CD44, CD45, CD105 were purchased from BD Pharmingen, San Diego, CA. Mouse anti-rat SM-MHC and FITCgoat anti-mouse IgG were purchased from Santa Cruz. 3-(4, 5-dimethylthiazol-2-yl)-2, 5-diphenyltetrazolium (MTT) was purchased from Sigma. Co. Trizol reagent was purchased from Invitrogen, USA. All other reagents were ultrapure grade.

\subsection{Cell Culture}

Rat MSCs were isolated from the femurs and tibias of male Sprague-Dawley rats $(90-100 \mathrm{~g})$ with a modified method originally described by Pittenger [3]. Briefly, bone marrow mononuclear cells were obtained by Percoll $(1.073 \mathrm{~g} / \mathrm{ml})$ density gradient centrifugation. The cells were seeded in Dulbecco's modified Eagle's medium-low glucose (DMEM-LG) supplemented with 10\% fetal bovine serum (FBS) and penicillin/streptomycin at $37^{\circ} \mathrm{C}$ in humified air with $5 \% \mathrm{CO} 2$. At $24 \mathrm{~h}$ after plating, nonadherent cells were removed by replacing medium. The antibiotic was removed after one media change. The medium was changed every $2-3$ days and the cells were passaged in $0.05 \%$ trypsin- $1 \mathrm{mM}$ EDTA. All the cells were used between passages 4 and 6 .

\subsection{Flow Cytometric Analysis}

Rat MSCs were phenotypically characterized by flow cytometry (Becton-Dickinson, San Jose, CA) by the method of Li [8]. The antibodies used in this study included FITC-conjugated or PE-conjugated antibodies CD14, CD29, CD34, CD44, CD45, CD105. To detect surface antigens, cells were collected and incubated (30 $\min$ at $48 \mathrm{C}$ ) with the respective antibody at a concentration previously established by titration. At least $1 \times 105$ cells for each sample were acquired and analyzed.

\subsection{Cell Differentiation Induction}

When the cultures of MSCs reached subconfluence, cells were washed twice with the medium and divided into two groups. In control group, the cells were cultured in basal DMEM medium (without FBS); in TGF- $\beta$ treatment group, the cells were incubated with $5,10 \mathrm{ng} / \mathrm{ml}$ TGF- $\beta$ in basal medium. Fresh TGF- $\beta$ was dissolved in phosphate buffered saline (PBS) and applied to cells. The cells in the two groups were incubated for 24 hours. The morphological changes of the cells were observed under phase contrast microscope (Nikon, Japan).

\subsection{Immunocytochemistry Assay}

After MSCs were treated in the two ways mentioned above for $24 \mathrm{~h}$, cells were fixed in $4 \%$ paraformaldehyde for $15 \mathrm{~min}$, blocked with normal goat serum for $20 \mathrm{~min}$ at room temperature (RT). Then, primary antibodies (mouse anti-rat SM-MHC) were added and incubated in a humid chamber over night. After washing with $0.1 \mathrm{M}$ phosphate buffered saline (PBS) three times, cells were incubated with appropriate secondary antibodies (FITCgoat anti-mouse IgG) for $30 \mathrm{~min}$ at $37^{\circ} \mathrm{C}$. After washing with $0.1 \mathrm{M}$ PBS, the samples were evaluated under inverted fluorescence microscope (Nikon, Japan).

\subsection{Cell Viability Assay}

Cells were seeded into 96-well plates and treated with or without TGF- $\beta$ for $24 \mathrm{~h}$, respectively. The viability of cells determined by using the method of MTT assay as described previously [9]. The light absorption was measured at $570 \mathrm{~nm}$ using Multiskan Spectrum (Thermo Labsystems). The viability (\%) was calculated by the formula as follow. Viability $(\%)=(\mathrm{OD}$ of control or treated group/OD of normal group $\times 100 \%$. The viability of normal group was presumed as $100 \%$.

\subsection{RNA Isolation and Semi-Quantitative Reverse-Transcription Polymerase Chain Reaction (RT-PCR)}

MSCs were treated in the two ways mentioned above for $24 \mathrm{~h}$. Semi-quantitative RT-PCR analysis was carried out as described previously [6]. Briefly, total RNA was isolated from cells using Trizol reagent, two microgram of the sample was reverse-transcribed using M-MLV reverse transcriptase (Promega, USA) according to the manufacturer's instructions. The PCR primer sequences are listed in Table 1. Semiquantitative analysis of mRNA expression was performed by using the Biorad software, using human glyceraldehyde-3-phosphate dehydrogenase (GAPDH) as a housekeeping gene.

\subsection{Statistical Analysis}

Data were expressed as mean $\pm \mathrm{SE}$ and accompanied by the number of experiments performed independently, and analyzed by t-test. Differences at $\mathrm{P}<0.05$ were considered statistically significant. 


\section{RESULTS}

\subsection{Immunophenotypic Characterization of Rat MSCs}

Rat MSCs isolated in this study were uniformly positive for CD29, CD44, CD105. In contrast, these cells were negative for other markers of the hematopoietic lineage CD14, CD34, the leukocyte common antigen CD45. Flow cytometry analyses showed that the MSC was a homogeneous cell population devoid of hematopoietic cells (Figure 1).

\subsection{TGF- $\beta$ Induced the SMC Differentiation of Rat MSCs}

Rat MSCs were exposed to $5,10 \mathrm{ng} / \mathrm{ml} \mathrm{TGF-} \beta 3$ respectively in the absence of serum. The treatment of MSCs with TGF- $\beta 3$ significantly changed the cell morphology. As show in Figure 2A, 24 hours after TGF- $\beta 3$ treatment, MSCs have a more spread out and myoblast-like morphology, and intracellular fibrous structures were visible. There were no obvious morphological changes in the control group. To confirm the SMC differentiation of these MSCs, we analyzed the expression of SM-MHC by immunocytochemistry. The cells treated with $5 \mathrm{ng} / \mathrm{ml}$ TGF- $\beta 3$ exhibited the positive SM-MHC (Figure 2B). The cells were treatment under the serum-free condition, thus we analyzed the cell viabilities. As shown in Figure 3 , the viabilities in the control and D609 treatment groups were decreased obviously at $24 \mathrm{~h}$, and are only about $60 \%$. There were no significant between control group and each experiment group $(P>0.05)$. These results shown that $10 \mathrm{ng} / \mathrm{ml}$ TGF- $\beta 3$ did not regulate the cell growth, but could induce the differentiation of MSCs.

To confirm the characters of these differentiated MSCs, the expression of SM22, SM- $\alpha$-actin, SM-MHC mRNA were examined. RT-PCR experiment results showed that at $24 \mathrm{~h}$, the MSCs treated with $5 \mathrm{ng} / \mathrm{ml}$ TGF- $\beta 3$ displayed weak expression of SM22, SM- $\alpha$-actin, SMMHC and $10 \mathrm{ng} / \mathrm{ml} \mathrm{TGF-} \beta 3$ induced the intensive increase of SM22, SM- $\alpha$-actin, SM-MHC in rat MSCs (Figure 4). In the control group, no expression of SM22, SM- $\alpha$-actin, SM-MHC were detected (Figure 4). These results showed that TGF- $\beta 3$ induced rat bone marrow-derived MSC differentiation into SMCs and increased the expression of contractile genes, such as SM22, SM-MHC, SM- $\alpha$-actin.

Table 1. The PCR primer sequence.

\begin{tabular}{cll}
\hline \multicolumn{1}{c}{ SM22 } & \multicolumn{1}{c}{ sense } & AGCCAGTGAAGGTGCCTGAGAAC \\
\hline \multirow{3}{*}{ SM- $\alpha$-actin } & antisense & TGCCCAAAGCCATTACAGTCCTC \\
& $\begin{array}{l}\text { sense } \\
\text { antisense }\end{array}$ & GAGAAGCCCAGCCAGTCG \\
SM-MHC & $\begin{array}{l}\text { sense } \\
\text { antisense }\end{array}$ & TGAGTCTCTGCGCTTCG \\
& GCAACAGAGTCCGCAAG \\
myocardin & sense & TCACCGCCTTAGCTCATACC \\
& antisense & CTGTCCCTCTGACCATTCTG \\
MRTF-A & sense & CTGACCCGAATGCTCCAACA \\
& antisense & CCAGGGCCATCTGCACTCTT \\
MRTF-B & sense & GTAGCCAGACCCTTGTTGCC \\
& antisense & TGTTTGGTGCCGAGTTTTG \\
GAPDH & sense & ATTCAACGGCACAGTCAAGG \\
& antisense & GCAGAAGGGGCGGAGATGA \\
\hline
\end{tabular}
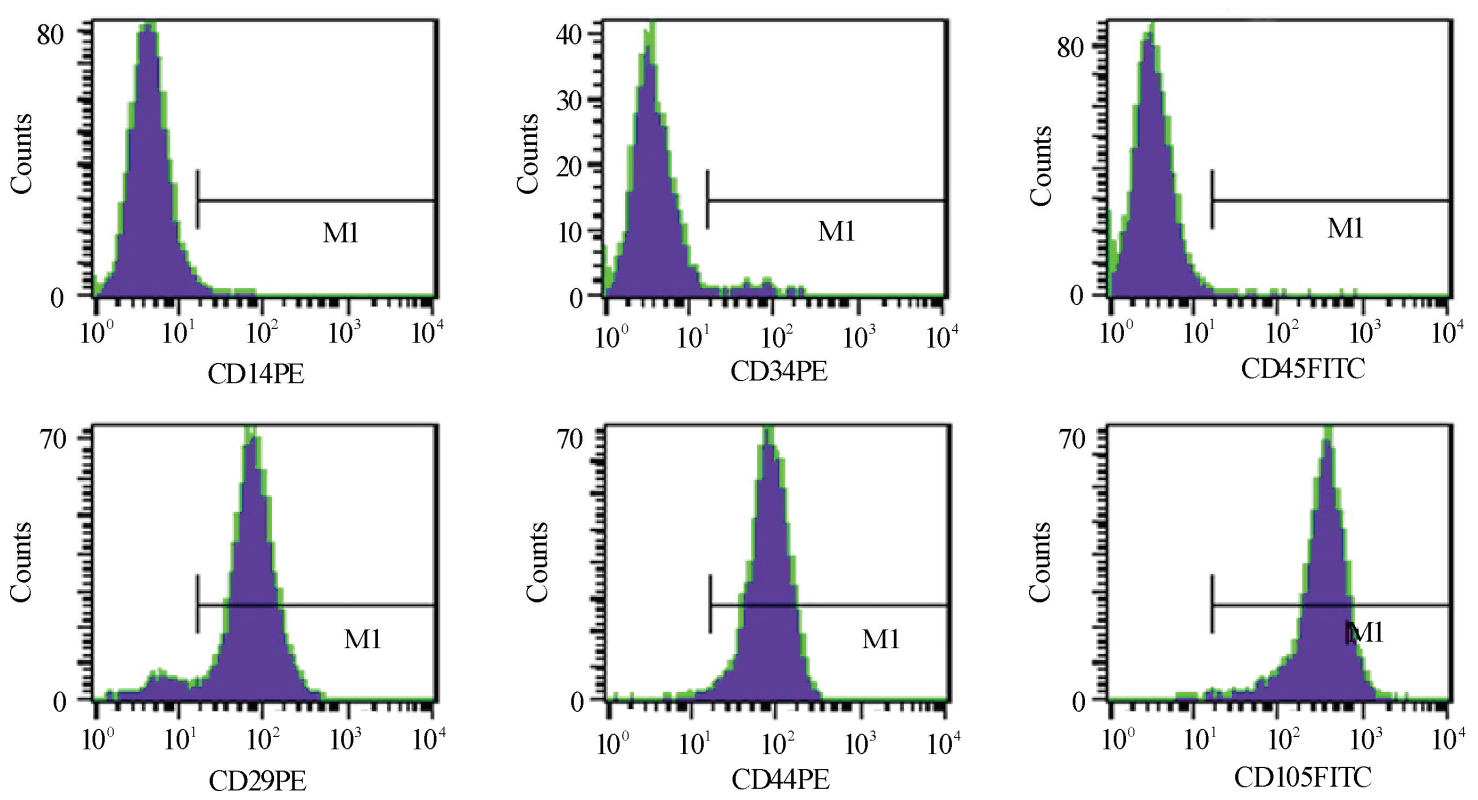

Figure 1. Immunophenotypic Characterixation of rat MSCs. the X-axis represents the cell number. 


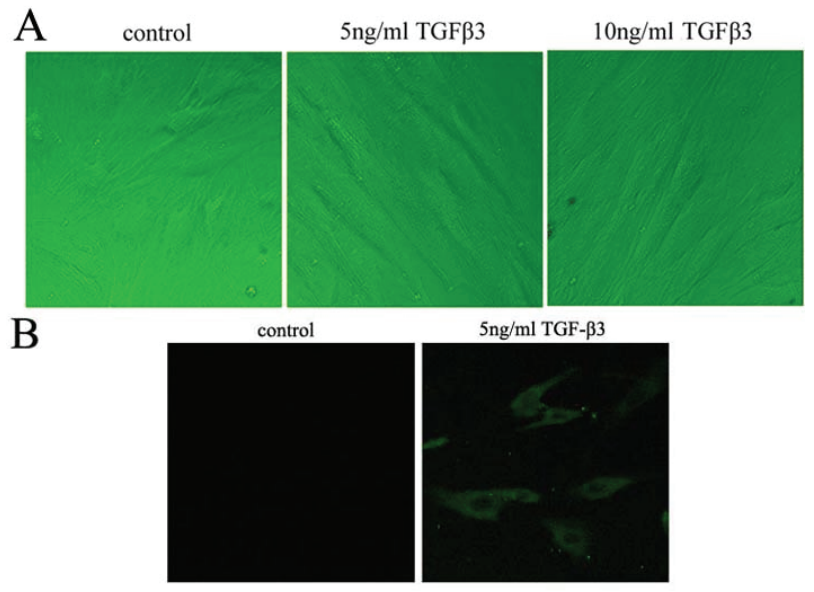

Figure 2. TGF- $\beta 3$ induce SMC differentiation of MSCs. (a) The morphological changes of rat MSCs; (b) The expression of SM-MHC in undifferentiated and differentiated cells. The cells were cultured in the serum-free medium with/without TGF- $\beta 3$ at $24 \mathrm{~h}$.

\subsection{Myocardin and MRTF-A were activated during the SMC differentiation of rat MSCs induced by TGF- $\beta$}

To investigate the role of MRTF family in the SMC differentiation of rat MSC, we detected the expression of myocardin, MRTF-A, MRTF-B by RT-PCR. It was observed that myocardin, MRTF-A were activated (Figure 5) during the SMC differentiation of rat MSCs induced by TGF- $\beta 3$, while no expression of MRTF-B was detected (data not shown). This result showed that myocardin, MRTF-A might contribute to the SMC differentiation of rat MSCs induced by TGF- $\beta 3$, but MRTF-B might not be implicated in this differentiation process.

\section{DISCUSSION}

SMCs are critical in development and postnatal life. SMCs have been implicated in vascular development as well as in a variety of cardiovascular diseases, including

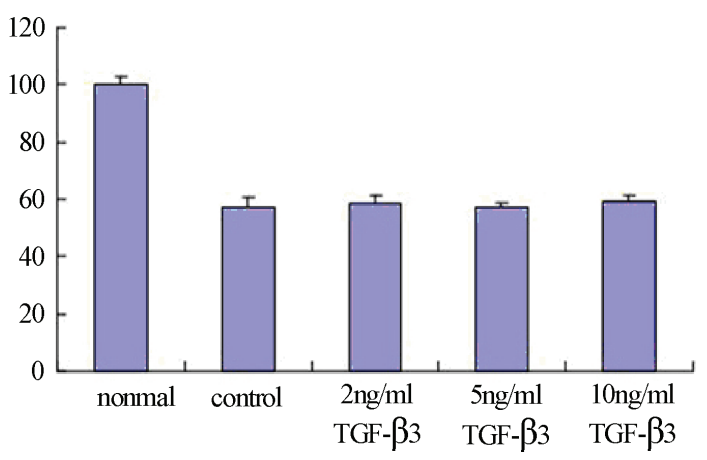

Figure 3. The viability of rat MSCs treated with TGF$\beta 3$. The cells were cultured in the serum-free medium with/ without TGF- $\beta 3$ at $24 \mathrm{~h}$. hypertension and atherosclerosis. Hence, studies aimed at the SMCs differentiation of rat MSCs are of great importance and will provide evidence for tissue engineering and therapeutic applications. Cellular transplantation therapy

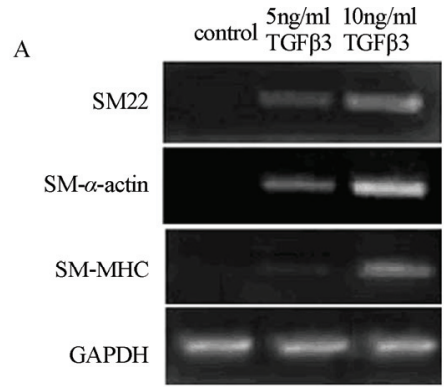

B

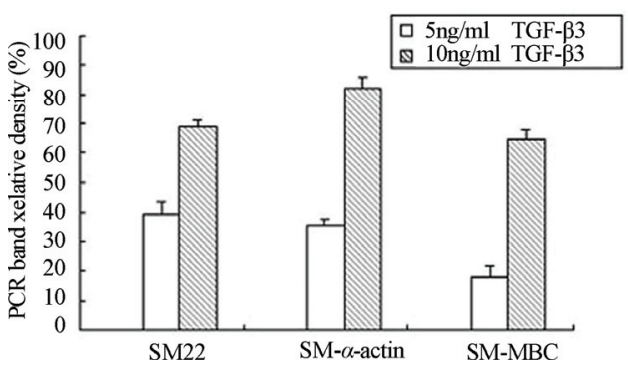

Figure 4. Expression of contractile genes in rat MSCs treated with TGF- $\beta 3$. (a) During the SMC differentiation of MSCs, the mRNA levels of SM22, SM- $\alpha$-actin, SM-MHC were elevated obviously. (b) Semiquantitative analysis of the contractile gene mRNA expression.
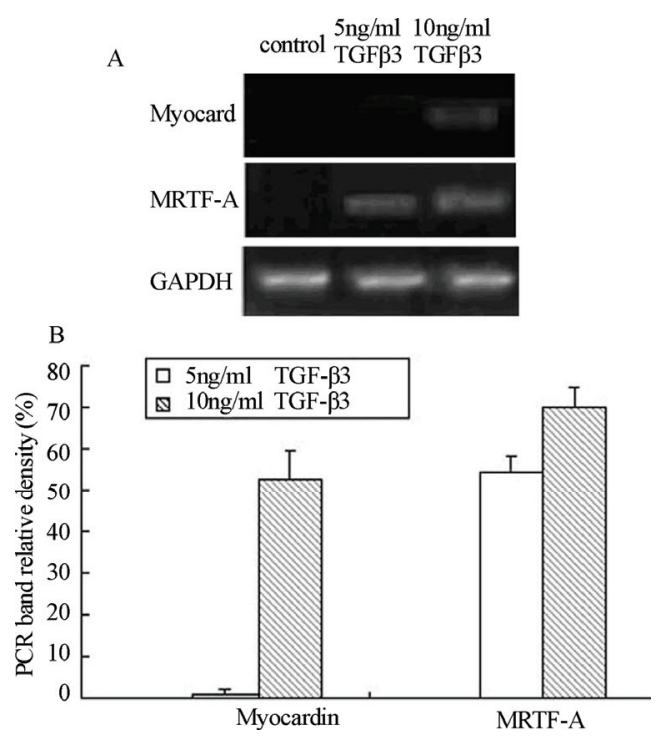

Figure 5. Expression of myocardin and MRTF-A in rat MSCs treated with TGF- $\beta 3$. (a) During the SMC differentiation of MSCs, myocardin and MRTF-A were activated. (b) Semiquantitative analysis of myocardin and MRTF-A mRNA expression. 
for the patients with cardiovascular diseases might possibly be achieved using the regenerated SMCs from autologous bone marrow cells in the near future. MSCs have great appeal for tissue engineering and therapeutic applications because of their general multipotentiality and relative ease of isolation. In vitro, MSCs have been shown to differentiate to SMCs in response to mechanical stress [10], growth factor, such as PDGF-BB and TGF- $\beta$ [11], and direct contact with vascular endothelial cells [12]. TGF- $\beta$ can contribute to development of SMCs from embryonic stem cells [13]. In this study, we found that TGF- $\beta 3$ could induce SMC differentiation of rat bone marrow-derived MSCs and increased the expression of contractile genes, such as SM22, SM- MHC, SM- $\alpha$-actin in MSCs.

However, the induction action of TGF- $\beta 3$ and the underlying molecular mechanisms involved in the differentiation of MSCs into SMCs are not well known. Myocardin is known to be a potent serum response factor (SRF) cofactor that plays important roles in regulating smooth muscle and cardiac muscle gene transcription [7]. It is reported that the expression of myocardin at the transcriptional level were increased during the SMC differentiation of hATSCs induced by SPC [6]. Taken together with previous experimental results, our findings are consistent with the idea that myocardin and MRTF-A is activated during the process of SMC differentiation in MSCs.

In summary, our results in this study showed that TGF- $\beta 3$ induced rat bone marrow-derived MSC differentiation into SMCs. TGF- $\beta 3$ increased the expression of contractile genes, such as SM22, smooth muscle-myosin heavy chain (SM-MHC), SM- $\alpha$-actin in MSCs. When TGF- $\beta 3$ induced MSCs differentiation into SMCs, myocardin and MRTF-A were activated. The data indicated that TGF- $\beta 3$ induced rat bone marrow-derived MSCs differentiation into SMCs by activating mypcardin and MRTF-A. Obviously, further studies are needed to clarify, for example, the interaction of myocardin and smad proteins as major intracellular mediators of TGF- $\beta$ signal pathways.

\section{REFERENCES}

[1] J. Y. Lai, C. Y. Yoon, J. J. Yoo, T. Wulf, and A. Atala. (2002) Phenotypic and functional characterization of in bivo tissue engineered smooth muscle from normal and pathological bladders. J. Urol., Sugar Land, 168, $1853-1857$.
[2] D. Orlic, J. Kajstura, S. Chimenti, I. Jakoniuk, S. M. Anderson, B. Li, et al. (2001) Bone marrow cells regenerate infarcted myocardium. Nature, 410, 701-705.

[3] M. F. Pittenger, A. M. Mackay, S. C. Beck, R. K. Jaiswal, R. Douglas, J. Mosca, et al., (1999) Multilineage potential of adult humanmesenchymal stem cells. Science, 284, 143-147.

[4] S. Davani, A. Marandin, N. Mersin, B. Royer, B. Kantelip, P. Herve, et al. (2003) Mesenchymal progenitor cells differentiate into an endothelial phenotype, enhance vascular density, and improve heart function in a rat cellular cardiomyoplasty model. Circulation, 108, II253-II258.

[5] B. Kinner, J. M. Zaleskas, M. Spector. (2002) Regulation of smooth muscle actin expression and contraction in adult human mesenchymal stem cells. Exp. Cell Res, 278, $72-83$.

[6] E. S. Jeon, H. J. Moon, M. J. Lee, H. Y. Song, Y. M. Kim, Y. C. Bae, et al., (2006) Sphingosylphosphorylcholine induces differentiation of human mesenchymal stem cells into smooth-muscle-like cells through A TGFbeta-dependent mechanism. J Cell Sci., 119, 4994-5005.

[7] G. C. Pipes, E. E. Creemers, E. N. Olson. (2006) The myocardin family of transcriptional coactivators: versatile regulators of cell growth, migration, and myogenesis. Genes Dev., 20, 1545-1556.

[8] C. D. Li, W. Y. Zhang, H. L. Li, X. X. Jiang, Y. Zhang, P. H. Tang, et al. (2005) Mesenchymal stem cells derived from human placenta suppress allogeneic umbilical cord blood lymphocyte proliferation. Cell Res., 15, 539-547.

[9] P. Price and T. J. McMillan. (1990) Use of the tetrazolium assay in measuring the response of human tumor cells to ionizing radiation. Cancer Res., 50, 1392-1396.

[10] N. Kobayashi, T. Yasu, H. Ueba, M. Sata, S. Hashimoto, M. Kuroki, et al. (2004) Mechanical stress promotes the epression of smooth muscle-like properties in marrow stromal cells. Exp. Hematol., 32, 1238-1245.

[11] J. J. Ross, Z. Hong, B. Willenbring, L. Zeng, B. Isenberg, E. H. Lee, et al. (2006) Cytokine-induced differentiation of multipotent adult progenitor cells into functional smooth muscle cells, J Clin Invest., 116, 3139-3149.

[12] S. G. Ball, A. C. Shuttleworth, C. M. Kielty. (2004) Direct cell contact influences bone marrow mesenchymal stem cell fate. Int. J. Biochem, Cell Biol., 36, 714-727.

[13] S. Sinha, M. H. Hoofnagle, P. A. Kingston, M. E. McCanna, G. K. Owens. (2004) Transforming growth factor- $\beta 1$ signaling contributes to development of smooth muscle cells from embryonic stem cells. Am. J. Physiol. Cell. Physiol., 287, C1560-C1568. 